\title{
Valor de la imagenología (TC y PET-TC) en el diagnóstico de la carcinomatosis peritoneal. Estudio preliminar
}

\author{
Dres. Álvaro Castro, Leandro Telles, Gabriela Rodríguez Cantera, \\ Daniel González, Fernando González, Luis Ruso Martínez
}

\section{Resumen}

En la evaluación de la carcinomatosis peritoneal (CP) la imagenología contribuye al diagnóstico preciso de compromiso peritoneal, aunque su eficacia es aún limitada. La tomografía computada (TC) es el estudio de imagen más utilizado y el PET-TC es cada vez más solicitado en la valoración de diferentes neoplasias. El objetivo del presente trabajo es evaluar el rendimiento de la TC y el PET-TC en el diagnóstico de CP en tumores digestivos y ginecológicos.

Material y método: estudio retrospectivo, observacional, de 11 casos operados de CP en los que previamente se había realizado TC o PET-TC, o ambos. Se analizan los resultados de los estudios imagenológicos por separado y en conjunto, y se comparan con los hallazgos intraoperatorios y anatomopatológicos.

Resultados: la TC fue concordante en 8 de 10 casos (S 78\%, E 100\%, VPP 100\%, VPN 33\%), mientras que el PET-TC lo fue en 9 de 11 pacientes (S 100\%, VPP 81\%). Al considerar en conjunto la TC y el PET-TC, la sensibilidad fue de $100 \%$ y el VPP de $82 \%$.

Conclusiones: en los resultados obtenidos se constata una subestimación de la CP en la imagenología a causa de las características de los nódulos y su patrón de captación. En el PET-TC, un SUV > 6 podría aumentar la probabilidad diagnóstica de carcinomatosis. Esta breve serie de casos permite sugerir que TC y el 18FGD PET-TC deben ser solicitados y analizados en forma conjunta para incrementar su rendimiento, aunque la exploración quirúrgica continúa siendo el método más eficaz para el diagnóstico y evaluación de la CP.

Palabras clave: Neoplasias peritoneales

Carcinoma

Peritoneo

Hipertermia inducida

Tomografía de emisión de positrones

Tomografía computarizada por rayos $X$

Key words: $\quad$ Peritoneal neoplasms

Carcinoma

Peritoneum

Hyperthermia, induced

Positron-emission tomography

Tomography, X-ray computed

Clínica Quirúrgica "1", Prof. Dr. Fernando González. Hospital Pasteur. Facultad de Medicina. Montevideo, Uruguay.

Clínica Quirúrgica "3", Prof. Dr. Daniel González. Hospital Maciel. Facultad de Medicina. Montevideo, Uruguay.

Recibido: 9/9/2019

Aceptado: $4 / 3 / 2020$ 


\section{Introducción}

La extensión peritoneal de los cánceres digestivos y ginecológicos es un factor de mal pronóstico y sobrevida limitada. Clásicamente estos pacientes han sido tratados con criterio paliativo, sin considerar mayormente la extensión de la enfermedad peritoneal. En estos casos la sobrevida ha sido breve, entre 3 a 6 meses.

En las últimas décadas se ha desarrollado una estrategia de tratamiento multimodal agresivo de la carcinomatosis peritoneal (CP), la cual se basa en la citorreducción quirúrgica (CR) completa y la quimioterapia intraperitoneal. Se ha demostrado que frente a una correcta selección de pacientes, con CP limitada, este tratamiento prolonga significativamente la sobrevida, e incluso en algunos casos puede lograr la curación ${ }^{(1,2)}$, siendo el principal factor pronóstico la extensión de la CP y la posibilidad de lograr la citorreducción macroscópica completa. La quimioterapia intraperitoneal está dirigida al tratamiento de la enfermedad microscópica residual tras la resección y se administra siguiendo diferentes estrategias, siendo la más utilizada actualmente la quimioterapia intraperitoneal hipertérmica (HIPEC), por su sigla en inglés: Hypertermic Intraperitoneal Chemotherapy. Este tratamiento aún está asociado a tasas variables de morbimortalidad ${ }^{(3,4)}$ y requiere seleccionar una población de pacientes con reserva funcional biológica para soportar esta cirugía y extensión de su enfermedad oncológica con expectativas de sobrevida prolongada. Está bien establecido que a menor extensión de la $\mathrm{CP}$, mejor será el pronóstico, mayor la efectividad de la CR+HIPEC y menor la morbimortali$\operatorname{dad}^{(5)}$.

En este contexto, el diagnóstico imagenológico constituye una importante herramienta para la detección y selección de casos con CP limitada, pasibles de tratamiento con óptimos resultados. Lamentablemente, la eficacia de las imágenes para el diagnóstico/evaluación de la CP en etapas tempranas es aún limitada y controversial, sobre todo en las formas micronodulares y en ciertas localizaciones de la cavidad abdominal.

La tomografía computada (TC) es el estudio de imagen más utilizado para el diagnóstico de tumores sólidos y el 18-FDG PET-TC es cada vez más solicitado en la evaluación de éstos, ya que junto a la resonancia nuclear magnética (RNM), hay evidencia de su alta precisión diagnóstica y ventajas funcionales y morfológicas para la caracterización tisular ${ }^{(6,7)}$.

En la TC, la CP puede evidenciarse mediante signos directos: la visualización de nódulos peritoneales o de una "galleta"/“torta" epiploica; así como por otros hallazgos más sutiles, como son: engrosamiento peritoneal o focal de la pared intestinal y la infiltración trabecular de la grasa del epiplón. También la presencia de ascitis es un signo indirecto de valor cuando se sospecha CP.

Las principales limitantes del diagnóstico tomográfico dependen de la técnica y de las lesiones. El estudio debe realizarse en un equipo multicorte y requiere un imagenólogo experiente para realizar un estudio dirigido a la detección de la $\mathrm{CP}$, a pesar de lo cual se constata una variación interobservador significativa. En cuanto a las lesiones peritoneales, la posibilidad de su detección depende de su tamaño, morfología y localización. La sensibilidad es baja para nódulos menores a $1 \mathrm{~cm}(9 \%$ a $24 \%$ ) y aumenta significativamente para lesiones mayores a $5 \mathrm{~cm}(59 \%$ a $90 \%)$. Los nódulos planos y dispuestos en forma de placa habitualmente son muy difíciles de evidenciar y pasan desapercibidos. Las lesiones localizadas en la pelvis y sobre el intestino delgado y su raíz son difíciles de diagnosticar. El rendimiento es mayor en flancos y fosas ilíacas ${ }^{(8,9)}$.

En el 18-FDG PET-TC se han descrito dos patrones de captación del radiotrazador. El patrón nodular o focal es el más frecuente y fácil de diagnosticar, son nódulos con clara hipercaptación, mientras que en el patrón difuso la captación es tenue, difícil de diferenciar de la captación fisiológica habitual del intestino; se vincula con lesiones peritoneales pequeñas y difusas, frecuentemente localizadas a lo largo del mesenterio e intestino delgado, así como a ascitis neoplásica escasa. Esta presentación es menos frecuente. Las principales limitantes del PET-TC son la posibilidad de falsos negativos debido a nódulos pequeños (especialmente menores de $5 \mathrm{~mm}$ ), nódulos sin hipermetabolismo, lesiones quísticas y tumores mucinosos, por su baja celularidad. También pueden existir errores de sobrediagnóstico, falsos positivos, debido a inflamación posquirúrgica, captación fisiológica del intestino, uréter o vejiga ${ }^{(10-12)}$.

\section{Objetivo}

El objetivo del estudio es analizar el valor clínico de la TC y el 18-FDG PET-TC en el diagnóstico de la CP por cáncer digestivo y ginecológico.

\section{Material y método}

Es un estudio retrospectivo realizado en un único centro, la Unidad de Carcinomatosis Peritoneal de la Clínica Quirúrgica “3” del Hospital Maciel, Montevideo, Uruguay.

Los criterios de inclusión fueron los siguientes: cáncer digestivo o de ovario con diagnóstico o sospecha de CP (sincrónica o metacrónica), evaluados por TC o 18-FDG PET-TC, o ambos, que fueron operados en forma consecutiva por CR + HIPEC y confirmado en informe anatomopatológico posoperatorio. 


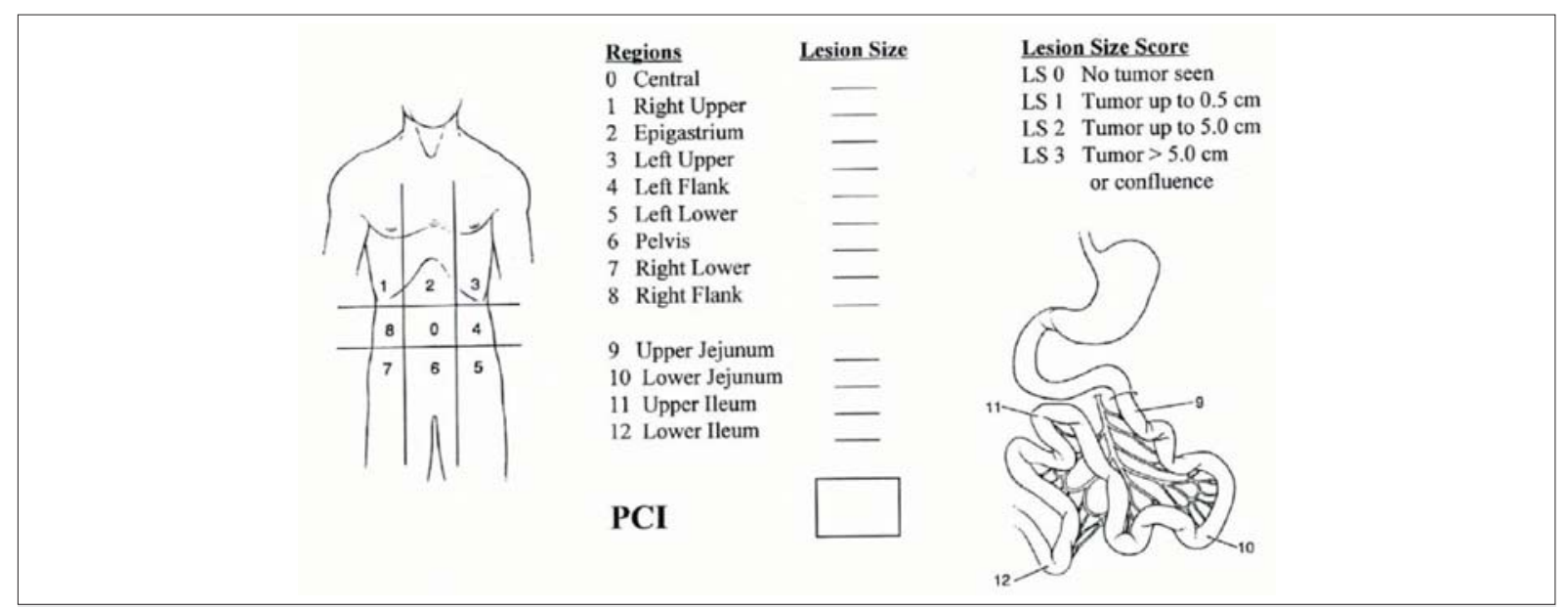

Figura 1. Hoja de registro para evaluación del ICP y la distribución topográfica de las lesiones. A los efectos de la correlación imagenológica las regiones 9 a 12 se incluyeron en las regiones 3, 0, 7 y 6, respectivamente.

El estudio consiste en analizar dichos estudios imagenológicos en relación con los hallazgos quirúrgicos de extensión lesional (índice de CP) y el reporte anatomopatológico. Se comparan los informes de la TC y el 18-FDG PET-TC versus la descripción operatoria y el informe anatomopatológico definitivo.

Las variables analizadas fueron en la TC: detección de CP (sí/no), tipo (focal única, focal múltiple, masiva, torta epiploica), extensión (número de cuadrantes de la cavidad abdomino-pélvica), topografía de las lesiones, tamaño de los nódulos visualizados (mayor y menor), presencia de ascitis, y en el 18-FDG PET-TC: detección de CP (sí/no), tipo (focal única, focal múltiple, masiva, difusa, torta epiploica), extensión (número de cuadrantes de la cavidad abomino-pélvica), topografía de las lesiones, tamaño de los nódulos visualizados (mayor y menor), captación máxima del radiotrazador expresada en standard uptake value ( SUV) o índice de captación estándar, que es un valor semicuantitativo que expresa la captación de la FDG en el tejido o lesión en relación con la dosis inyectada, y se define como el cociente entre la concentración tisular de radiofármaco y la dosis inyectada dividida por el peso corporal en gramos. Se considera un umbral de SUV máximo (SUVmáx) superior a 2,99 para indicar malignidad ${ }^{(13)}$.

De la descripción operatoria se utilizaron como indicadores: la detección de CP (sí/no), su extensión (según PCI y número de cuadrantes afectados), topografía de las lesiones, tamaño de los nódulos (mayor y menor), presencia de ascitis (sí/no) y estimación de su magnitud (escasa, moderada, severa).

Del informe anatomopatológico fueron analizados: existencia de $\mathrm{CP}$ en las lesiones resecadas (sí/no), grado de diferenciación tumoral (bien moderadamente dife- renciado, mal diferenciado) y si se trataba de un tumor mucinoso (sí/no).

La extensión de la CP en los estudios de imagen fue medida adaptando el esquema de distribución y cálculo de ICP, según se observa en la figura 1. El PCI realizado durante la exploración operatoria divide el abdomen en 13 regiones (numeradas de 0 a 12). Las primeras nueve zonas (numeradas 0 a 8) se corresponden con la división clínica clásica del abdomen, las cuales también se pueden diferenciar topográficamente en los estudios de imagen. Por lo tanto, en estos nueve cuadrantes existe correspondencia topográfica entre la imagen y la exploración quirúrgica. Sin embargo, en las últimas cuatro zonas del PCI que dividen el mesenterio e intestino delgado en yeyuno proximal (zona 9), yeyuno distal (zona 10), íleon proximal (zona 11), íleon distal (zona 12), resulta muy difícil -en los estudios de imagen-discriminar las lesiones con exactitud debido a la superposición imagenológica, movilidad y variaciones que puede presentar el intestino delgado. Por tanto, para poder comparar la extensión de la $\mathrm{CP}$ detectada por cirugía, según el PCI, con la detectada por los estudios de imagen, se debe realizar una adaptación del número de zonas del PCI. En nuestro caso, dividimos siempre el abdomen en los nueve cuadrantes clásicos (zonas 0 a 8 del PCI), tanto para la imagen como para la cirugía. Las zonas intestinales del PCI quirúrgico (numeradas de 9 a 12), cuando eran positivas, se incluyeron dentro de los correspondientes cuadrantes; luego, con un criterio de proximidad, la zona 9 (yeyuno proximal) se incluyó en la 3 (hipocondrio izquierdo), la zona 10 (yeyuno distal) en la 0 (región umbilical), la zona 11 (íleon proximal) en la 6 (hipogastrio), la zona 12 (íleon distal) en la 7 (fosa ilíaca derecha), para así poder establecer una comparación image- 
Tabla 1. Valoración de extensión de la CP por TC en comparación con exploración quirúrgica y anatomía patológica.

\begin{tabular}{lccc}
\hline Caso & TC & Cirugía + AP & CORRELACIÓN \\
\hline 1 & 9 & $9(\mathrm{PCl} 37)$ & ACORDE \\
2 & 2 & $2(\mathrm{PCl} 4)$ & ACORDE \\
3 & 1 & $5(\mathrm{PCl} 10)$ & SUBESTIMÓ \\
4 & 1 & $1(\mathrm{PCl} 3)$ & ACORDE \\
5 & 1 & $1(\mathrm{PCl} 3)$ & ACORDE \\
6 & 0 & $1(\mathrm{PCl} 1)$ & SUBESTIMÓ \\
7 & 1 & $9(\mathrm{PCl} 37)$ & SUBESTIMÓ \\
8 & 3 & $8(\mathrm{PCl} 20)$ & SUBESTIMÓ \\
9 & No TC & No TC & No TC \\
10 & 0 & 0 & ACORDE \\
11 & 0 & $9(\mathrm{PCl} 37)$ & SUBESTIMÓ \\
\hline
\end{tabular}

nológica - quirúrgica de la extensión de la $\mathrm{CP}$ en nueve cuadrantes.

Además, para nuestro estudio, dividimos la $\mathrm{CP}$ en limitada o extensa, definiendo como CP limitada a la que afecta hasta dos cuadrantes del abdomen y/o presentaba un PCI de hasta 6 y extensa por encima de estos indicadores.

\section{Resultados}

Se recolectaron los datos de 11 pacientes consecutivos que cumplían con los criterios de inclusión, comprendidos entre junio de 2013 y setiembre de 2017. Diez mujeres y un hombre. La mediana de edad fue de 59 años (rango: 29-75).

Los cánceres primitivos fueron cinco tumores de ovario y seis tumores digestivos (cuatro de colon, uno de intestino delgado, uno de páncreas). En dos casos eran tumores mucinosos, uno de ovario y otro de colon.

La CP fue sincrónica en dos casos. En los restantes nueve fue matacrónica al tumor primario, que ya había sido resecado previamente.

De los 11 pacientes, 10 tenían TC y todos PET-TC. Las TC se realizaron en diferentes centros, equipos y por diferentes técnicos, sin un protocolo uniforme preestablecido, ya que la mayoría de los casos son pacientes derivados desde otras instituciones. En todos los casos la TC se realizó con contraste intravenoso. Por el contrario, el 18-FDG PET-TC se realizó siempre en el Centro Uruguayo de Imagenología Molecular (CUDIM).
Tabla 2. Valoración de extensión de la CP por PET-TC en comparación con la exploración quirúrgica más anatomía patológica.

\begin{tabular}{lccc}
\hline Caso & PET-TC & Cirugía $+A P$ & CORRELACIÓN \\
\hline 1 & 9 & $9(\mathrm{PCI} 37)$ & ACORDE \\
2 & 2 & $2(\mathrm{PCl} 4)$ & ACORDE \\
3 & 1 & $5(\mathrm{PCl} 10)$ & SUBESTIMÓ \\
4 & 2 & $1(\mathrm{PCl} 3)$ & SOBRESTIMÓ \\
5 & 2 & $1(\mathrm{PCl} 3)$ & SOBRESTIMÓ \\
6 & 1 & 0 & SOBRESTIMÓ \\
7 & 2 & $9(\mathrm{PCI} 37)$ & SUBESTIMÓ \\
8 & 8 & $8(\mathrm{PCI} 20)$ & ACORDE \\
9 & 9 & 9 & ACORDE \\
10 & 2 & 0 & SOBRESTIMÓ \\
11 & 6 & $9(\mathrm{PCI} 37)$ & SUBESTIMÓ \\
\hline
\end{tabular}

LA TC detectó la CP en ocho de diez casos. O sea, presentó dos casos falsos negativos Su sensibilidad para el diagnóstico de CP fue de $78 \%$, especificidad de $100 \%$, valor predictivo positivo (VPP) de $100 \%$ y valor predictivo negativo (VPN) de 33\%. Los dos casos falsos negativos fueron: 1) el caso 6 , paciente portador de un primitivo pancreático, quien presentó un único nódulo menor a $1 \mathrm{~cm}$ durante la cirugía; 2) el caso 11, que en la cirugía presentó $\mathrm{CP}$ extensa a nódulos pequeños distribuidos mayormente en placas planas.

En cuanto a la extensión de la $\mathrm{CP}$, según el número de cuadrantes comprometidos, la TC fue acorde con la exploración quirúrgica y confirmada por el estudio histológico en cinco casos, mientras que en los otros cinco subestimó la extensión de la misma (tabla 1). El nódulo de menor tamaño detectado por TC fue de $1,9 \mathrm{~cm}$; con una mediana de $3,6 \mathrm{~cm}(1,9-5 \mathrm{~cm})$.

Para el diagnostico de ascitis, la TC fue concordante con los hallazgos intraoperatorios en 7 de 10 casos, presentando dos falsos negativos y un falso positivo. $\mathrm{Su}$ sensibilidad fue de $50 \%$, especificidad de $83 \%$, VPP $66,6 \%$ y VPN $71 \%$. Los dos casos falsos negativos fueron: 1) caso 7, en la cirugía se encontró ascitis severa/ abundante, pero el tiempo transcurrido entre la TC y la cirugía fue mayor a tres meses, con una rápida progresión de la enfermedad; 2) caso 11, en la cirugía se detectó ascitis muy escasa. El caso falso positivo fue el caso 10, la TC informó lámina de líquido en pelvis que no se constató durante la cirugía. 
El PET-TC detectó correctamente la $\mathrm{CP}$ en 9 de 11 casos, presentando dos casos falsos positivos. No tuvo falsos negativos. La sensibilidad para el diagnóstico de CP fue de $100 \%$ y el VPP de $81 \%$. La especificidad y VPN no pudieron ser establecidos debido a que en esta serie, pequeña, no se presentaron casos falsos ni verdaderos negativos. Los dos casos falsos positivos (el PET-TC informó CP que no fue comprobada en la exploración quirúrgica ni por estudio histológico posoperatorio) fueron: 1) el caso 6, cáncer de cola de páncreas, el PET-TC informó un nódulo peritoneal sincrónico de 1 cm hipercaptante (SUV 5,3) en flanco izquierdo sobre el peritoneo parietal anterior, el cual no se confirmó posteriormente en la cirugía; 2) el caso 10, cáncer de colon en el cual el PET-TC informó dos nódulos sincrónicos hipercaptantes con SUV máximo 5,8, pequeños (el mayor de $1,7 \mathrm{~cm}$ ) en el mesenterio, los cuales no fueron confirmados en la cirugía $(\mathrm{PCI}=0$ ).

En cuanto a la extensión de la $\mathrm{CP}$, según el compromiso del número de cuadrantes, el PET-TC fue acorde al diagnóstico intraoperatorio en cuatro casos. En tres casos subestimó la extensión y en cuatro casos la sobrestimó (tabla 2). El nódulo de menor tamaño detectado fue de $0,6 \mathrm{~cm}$, con una mediana de 2,2 $(0,6-10 \mathrm{~cm})$. En los dos casos de tumores mucinosos el PET-TC fue positivo con captación del radiotrazador.

En cuanto a la intensidad de captación del radiotrazador en las lesiones peritoneales, la mediana del SUV fue de 7,6, con un rango de 4,4 a 26. Los dos casos en que el PET-TC falló en el diagnóstico al informar nódulos de CP que no existían, casos falsos positivos, tuvieron un SUV menor a 6. Por el contrario, 7 de los 8 verdaderos positivos tenían un SUV mayor a 6 .

Al combinar los resultados de la TC y el PET-TC en cada paciente, se obtiene que de los 11 casos, hubo nueve aciertos dados por verdaderos positivos y dos errores dados por falsos positivos. La combinación de ambos estudios de imagen da una sensibilidad de $100 \%$ y un VPP de $82 \%$ para el diagnóstico de CP. La especificidad y VPN no pudieron ser valorados debido a la ausencia de casos falsos y verdaderos negativos. De los nueve casos verdaderos positivos, en siete ambos estudios fueron positivos para el diagnóstico de $\mathrm{CP}$, posteriormente comprobada en la cirugía. En dos casos solo el PET-TC hizo el diagnóstico preoperatorio, mientras que la TC había sido negativa para CP. Los dos casos de error fueron falsos positivos debidos al PET-TC, como ya se comentó. La TC no había evidenciado nódulos peritoneales en ninguno de ellos.

\section{Discusión}

El diagnóstico imagenológico de la $\mathrm{CP}$, cuando es escasa o pequeña, es habitualmente difícil y no existe un es- tudio único de imagen con alta sensibilidad y especificidad para ello.

El estudio más utilizado para su valoración y diagnóstico ha sido la TC. El PET/TC con 18- FDG se utiliza ampliamente como una potente modalidad de evaluación en medicina nuclear oncológica, no solo para detectar tumores, sino también para estadificación y para monitorización de la terapia ${ }^{(14)}$, basado en que proporciona información sobre la extensión del tumor y es particularmente útil para la identificación de metástasis a distan$\mathrm{cia}^{(15)}$. Esto ha generado la interrogante acerca de si es el mejor estudio para la detección de nódulos peritoneales, porque aún algunos expertos consideran la TC toraco abdominal suficiente para una estadificación preoperatoria de rutina y dejan la PDG PET-T, la laparotomía y la laparoscopía como herramientas complementarias ${ }^{(16)}$.

En la utilización de SUV como variable de decisión se considerara que existen numerosas causas de absorción de FDG en procesos benignos observados en imágenes PET y en tumores con baja celularidad, como aquellos con alto componente de mucina. De hecho, el grado de absorción de FDG está relacionado con la tasa metabólica celular y el número de transportadores de glucosa y su acumulación en los tumores se debe, en parte, a un mayor número de transportadores de glucosa en las células malignas. Aun así, la FDG no es específica para las neoplasias: existe una situación similar en la inflamación, las células inflamatorias activadas demuestran una mayor expresión de transportadores de glucosa.

Por tanto, existe un interés creciente en mejorar la especificidad de FDG-PET en pacientes con cáncer. Los estudios preliminares mostraron que en varios tipos tumorales la captación de FDG continúa aumentando durante horas después de la inyección radiofarmacéutica y esta diferencia en el transcurso del tiempo de la captación de FDG podría ser útil para mejorar la precisión de la PET con el fin de distinguir las lesiones benignas de las malignas, lo cual ha generado procedimientos de medición tardía (dual point) capaces de diferenciar el tejido inflamatorio del tejido neoplásico. Se postula que el tejido inflamatorio reduzca la absorción de FDG a medida que pasa el tiempo, mientras que se supone que la absorción en las lesiones neoplásicas está aumentando ${ }^{(14)}$.

Turlakow $^{(10)}$ evaluó la eficacia diagnóstica del 18-FDG PET en la CP y lo compararon con TC. Diseñaron un estudio retrospectivo que incluyó 88 pacientes con diversos tumores (48 cánceres gástricos, 13 de ovario, 21 mesoteliomas, 6 cánceres adrenales). Todos los casos contaban con TC y PET, en los cuales se buscó evidencia de CP. Como "patrón de referencia" para valorar sus resultados, se consideró el diagnóstico de CP por histología, citología o imagenológico evolutivo durante la enfermedad. Los estudios de imagen sospecha- 
ron $\mathrm{CP}$ en 24 de los 88 casos, la cual fue posteriormente confirmada en 23 (27\%). La TC tuvo una sensibilidad diagnóstica de $43 \%$ y un VPP de $100 \%$. El PET obtuvo una sensibilidad de $57 \%$ y un VPP de $93 \%$. Al considerar los dos estudios en conjunto, la sensibilidad subió a $78 \%$, y el VPP se mantuvo alto, en $95 \%$. En menos de la mitad de casos los dos estudios aislados informaban CP. En casi el $60 \%$ de los casos positivos, solo uno de los dos estudios había detectado CP. Por tanto, los autores concluyen que el 18-FDG PET mejora el diagnóstico de CP cuando se combina con la imagen convencional (TC). En nuestro estudio se confirma el mismo hecho, ya que la combinación del PET-TC y la TC logró aumentar la sensibilidad diagnóstica de esta última desde $78 \%$ a $100 \%$, con un VPP de $82 \%$.

Dromain ${ }^{(17)}$ comparó la TC vs PET-TC para diagnóstico y extensión de la CP. Realizó un estudio retrospectivo con 30 pacientes con sospecha de CP metacrónica por tumores digestivos. Todos contaban con TC y PET-TC y todos fueron a cirugía (CRS + HIPEC). Se verificaron dos casos falsos positivos (CP que no se constató en la cirugía). En nuestra breve serie, tuvimos dos falsos positivos del PET-TC, lo cual se debe atribuir a las características de la $\mathrm{CP}$, a nódulos pequeños, limitada, sincrónica y con un SUV de 5,3 y 5,8.

En el estudio mencionado, la TC tuvo una sensibilidad de $82 \%$, similar a la que obtuvimos en nuestros casos (78\%). El PET-TC obtuvo una sensibilidad inferior a la TC de solo $57 \%$, lo cual contrasta con lo comunicado por Turlakow ${ }^{(10)}$ y con nuestra propia serie.

Ambos estudios, la TC y el PET-TC, tuvieron una baja a moderada correlación con la exploración quirúrgica en cuanto a la extensión de la $\mathrm{CP}$, subestimándola en $70 \%$ a $80 \%$ de casos. Este hecho se reproduce en nuestra serie y ninguno de los dos estudios mostró ser efectivo en la valoración real de extensión de la $\mathrm{CP}$. Al igual que en nuestra experiencia la relación TC/cirugía fue en solo $50 \%$ de los casos y el PET-TC/cirugía se correlacionó menos aun $(36,6 \%)$. Posteriormente, Berthelot ${ }^{(18)}$, en otro estudio retrospectivo, evaluó el PET-TC para el diagnóstico y extensión de CP. Comparó sus resultados con la TC y la exploración quirúrgica, tomando como patrón de referencia para el diagnóstico efectivo de $\mathrm{CP}$ el informe anatomopatológico posoperatorio. Analizaron 28 casos con sospecha imagenológica de $\mathrm{CP}$ por cánceres digestivos (17 colorrectales, 1 gástrico), de ovario (9) y uno mesotelioma. De los 28 casos, en 23 (82\%) se confirmó la CP por anatomía patológica. La TC tuvo una sensibilidad muy baja, de solo $30 \%$, aunque esto pudo deberse a la metodología del estudio, mientras que el PET-TC tuvo una sensibilidad de $82,6 \%$, especificidad de $60 \%$, VPP $90 \%$ y VPN $50 \%$. A diferencia del resto de los estudios y de nuestra propia serie, el PET-TC demostró una buena concordancia en el cálculo de la extensión de la CP en relación con la exploración quirúrgica y a la histología posoperatoria. Los autores concluyeron que en pacientes con riesgo para $\mathrm{CP}$, este estudio tiene excelente resultados tanto para el diagnóstico positivo como para la estimación de la extensión de la CP.

Diferentes publicaciones ${ }^{(19,20)}$ han reportado que el PET-TC disminuye su sensibilidad diagnóstica frente a lesiones mucinosas debido a la inactividad metabólica de la mucina y a la baja celularidad de estos tumores, aunque otros autores no han encontrado diferencias significativas de captación entre lesiones mucinosas y no mucinosas $^{(11)}$ : En nuestro estudio hubo dos tumores mucinosos. En ambos casos el PET-TC detectó las lesiones peritoneales que posteriormente se constataron en la cirugía. Debido a lo pequeño de nuestra muestra, necesitamos recolectar más casos para valorar el rendimiento del PET-TC frente a este tipo de tumores.

Recientemente, Rubini ${ }^{(21)}$ comunica un estudio retrospectivo sobre el rol del 18FDG PET/TC en el diagnóstico de $\mathrm{CP}$ metacrónica en cáncer de ovario, comparando su rendimiento con el de la TC y el marcador tumoral Ca-125. Analizaron 79 casos de cáncer de ovario estadio III o IV, ya resecados, y obtuvieron un buen rendimiento, con una sensibilidad de $85 \%$, especificidad de $92 \%$ y exactitud diagnóstica de $88,6 \%$. El SUV promedio en las lesiones peritoneales fue de 7,7, y al analizar que la intensidad de captación aumenta la eficacia diagnóstica, los autores encontraron que con un SUV mayor a 5 la exactitud es de 78\%. Estos datos son acordes a los de nuestro trabajo, ya que obtuvimos un SUV con mediana de 7,6, mientras que los dos casos falsos positivos tenían un SUV menor a 6. Por el contrario, casi todos los verdaderos positivos ( 7 de 8 ) tenían un SUV mayor a 6. Por tanto, es racional establecer que un punto de corte en SUV de 6 o mayor aumenta la probabilidad de que un nódulo peritoneal hipercaptante sea efectivamente neoplásico, disminuyendo la probabilidad de falsos positivos del estudio.

Asimismo, $\mathrm{Li}^{(22)}$, en una reciente revisión, muestra que la sensibilidad combinada de PET/CT (84\%) fue significativamente mayor que la de PET (60\%), y la especificidad combinada de PET (98\%) fue notablemente mayor que la de PET/CT (94\%). Sobre una base por lesión, la sensibilidad y especificidad agrupadas de $\mathrm{PET} / \mathrm{CT}$ fueron $87 \%$ y $95 \%$, respectivamente. Solo en un estudio de PET por lesión, su sensibilidad es $65,8 \%$ y la especificidad de $94,1 \%$ y concluye que PET y $\mathrm{PET} / \mathrm{CT}$ son potentes técnicas de imagen para la detección y caracterización de PC y sugiere utilizar el PET/TC como herramienta de detección y la PET como herramienta de diagnóstico. 
El presente estudio tiene varias limitantes, ser retrospectivo, basado en informes imagenológicos y no en la revisión de imágenes por expertos, tener un bajo número de pacientes y utilizar el SUV como variable de corte diagnóstica. No encontramos otros trabajos nacionales sobre este tema y la literatura internacional también muestra series breves y retrospectivas. Se necesitan trabajos cooperativos entre imagenólogos y clínicos para avanzar en el tema, incluyendo una técnica muy prometedora de RNM para detectar metástasis peritoneales, como es la imagen de difusión ponderada (Diffusionweighted MRI [DWI] $)^{(23)}$.

\section{Conclusiones}

Considerando que el diagnóstico preciso de $\mathrm{CP}$ por imágenes es aún limitado, especialmente en etapas tempranas, se estudiaron 11 pacientes portadores de $\mathrm{CP}$ mediante TC y PETC-TC, verificándose que ambos estudios en forma independiente tienen aceptable eficacia, aunque su sensibilidad diagnóstica aumenta al combinarlos. Sus resultados son limitados, porque se constata una subestimación de la CP en la imagenología a causa de las características de los nódulos y su patrón de captación. En el PET-TC, un SUV > 6 podría aumentar la probabilidad diagnóstica de carcinomatosis.

Del análisis comparativo de las imágenes con la cirugía, se puede inferir que actualmente el método con mejores resultados para medir la existencia, extensión y topografía de la $\mathrm{CP}$ es la exploración quirúrgica. Esta breve serie de casos permite sugerir que la TC y el 18FGD PET-TC deben ser solicitados y evaluados en forma conjunta para incrementar su rendimiento.

\section{Summary}

Imagenology contributes to the accurate diagnosis of peritoneal compromise in the assessment of peritoneal carcinomatosis, although its effectiveness is still limited. CT is the most frequently used study and PET/CT is graduallly more requested in the assessment of different neoplasms. This study aims to assess performance of $\mathrm{CT}$ and PET/CT for diagnosis of peritoneal carcinomatosis in digestive and gynecological tumours.

Method: retrospective, observational study of 11 cases operated on peritoneal carcinomatosis after a CT and/or PET/CT had been performed. The results of both image studies were analysed separately and altogether and they were subsequently compared to intraoperative and pathology studies.

Results: CT matched findings in 8 out of 10 cases (sensitivity $78 \%$, specificity $100 \%$, positive predictive value $100 \%$, negative predictive value $33 \%$ ), whereas PET/TC matched in 9 out of 11 patients (sensitivity
$100 \%$, PPV $81 \%$ ). When considered together, sensitivity was $100 \%$ y el PPV de $82 \%$.

Conclusions: the results of the study confirm imagenology studies overestimate peritoneal carcinomatosis based on the characteristics of nodules and its uptake pattern. In CT/PET, SUV $>6$ could increase the probability of diagnosing carcinomatosis. This small series of cases enables our suggesting CT and 18F-FDG PET-TC to be requested and analysed together to increase its efficiency, although surgical exploration continues to be the most effective method to diagnose and assess peritoneal carcinomatosis.

\section{Resumo}

$\mathrm{Na}$ avaliação da carcinomatose peritoneal $(\mathrm{CP})$ as técnicas de imagem contribuem ao diagnóstico preciso do compromisso peritoneal, embora sua eficácia ainda seja limitada. A tomografia computadorizada (TC) é o estudo de imagens mais utilizado e a tomografia computadorizada por emissão de pósitrons (PET-TC) é cada vez mais solicitado na avaliação de diferentes neoplasias. O objetivo do presente trabalho, é avaliar o rendimento da TC e do PET-TC no diagnóstico de $\mathrm{CP}$ em tumores digestivos e ginecológicos.

Material e método: estudo retrospectivo, observacional de 11 casos de carcinomatose peritoneal operados nos quais previamente se havia realizado $\mathrm{TC}$ e/ou PET-TC. Os resultados de ambos estudos foram estudados isoladamente e em conjunto e foram comparados com os achados intraoperatórios e histopatológicos.

Resultados: a TC foi concordante em 8 de 10 casos (S 78\%, E 100\%, VPP 100\%, VPN 33\%), e o PET-TC em 9 de 11 pacientes (S 100\%, VPP 81\%). A sensibilidade foi de $100 \%$ e o VPP de $82 \%$ quando ambos estudos foram analisados conjuntamente.

Conclusoes: observa-se nos resultados uma subestimação da $\mathrm{CP}$ nas técnicas de imagem, devido às características dos nódulos e seu padrão de captação. No PET-TC, um SUV $>6$ poderia aumentar a probabilidade de diagnosticar carcinomatose. Esta breve série de casos permite sugerir que a TC e o 18FGD PET-TC devem ser solicitados e analisados em forma conjunta para aumentar o rendimento. No entanto a exploração cirúrgica continua sendo o método mais eficaz para o diagnóstico e avaliação da CP.

\section{Bibliografía}

1. Verwaal VJ, van Ruth S, de Bree E, van Slooten GW, van Tinteren H, Boot H, et al. Randomized trial of cytoreduction and hyperthermic intraperitoneal chemotherapy versus systemic chemotherapy and palliative surgery in patients with peritoneal carcinomatosis of colorectal cancer. J Clin Oncol 2003; 21:3737-43. 
2. Glehen O, Kwiatkowski F, Sugarbaker PH, Elias D, Levine EA, De Simone M, et al. Cytoreductive surgery combined with perioperative intraperitoneal chemotherapy for the management of peritoneal carcinomatosis from colorectal cancer: a multi-institutional study. J Clin Oncol 2004; 22:3284-92.

3. Stephens AD, Alderman R, Chang D, Edwards GD, Esquivel J, Sebbag G, et al. Morbidity and mortality analysis of 200 treatments with cytoreductive surgery and hyperthermic intraoperative intraoperitoneal chemotherapy using the coliseum technique. Ann Surg Oncol 1999; 6:790-6.

4. Verwaal VJ, van Tinteren H, Ruth SV, Zoetmulder FA. Toxicity of cytoreductive surgery and hyperthermic intra-peritoneal chemotherapy. J Surg Oncol 2004; 85:61-7.

5. Sugarbaker PH. Early intervention for treatment and prevention of colorectal carcinomatosis: a plan individualized care. Surg Oncol Clin N Am 2012; 21:689-703.

6. Kim SJ, Lee SW. Diagnostic accuracy of ${ }^{18}$ F-FDG PET/CT for detection of peritoneal carcinomatosis; a systematic review and meta-analysis. Br J Radiol 2018; 91(1081):20170519.

7. Sanli Y, Turkmen C, Bakir B, Iyibozkurt C, Ozel S, Has D, et al. Diagnostic value of PET/CT is similar to that of conventional MRI and even better for detecting small peritoneal implants in patients with recurrent ovarian cancer. Nucl Med Commun 2012; 33:509-15.

8. de Bree E, Koops W, Kroger R, van Ruth S, Witkamp AJ, Zoetmulder FA. Peritoneal carcinomatosis colorectal or appendiceal origin: correlation of preoperative $\mathrm{CT}$ with intraoperative findings and evaluation of interobserver agreement. Surg Oncol 2004; 86(2):64-73.

9. Jacquet P, Felinek JS, Steves MA, Sugarbaker PH. Evaluation of computed tomography in patients with peritoneal carcinomatosis. Cancer 1993; 72:1631-6.

10. Turlakow A, Yeung HW, Salmon AS, Macapinlac HA, Larson SM. Peritoneal carcinomatosis: role of (18)F-FDG PET. J Nucl Med 2003; 44:1407-12.

11. Suzuki A, Kawano T, Takahashi N, Lee J, Nakagami Y, Miyagi E, et al. Value of $18 \mathrm{~F}$-FDG PET in the detection of peritoneal carcinomatosis. Eur J Nucl Med Mol Imaging 2004; 31:1413-20.

12. Tanaka T, Kawai Y, Kanai M, Taki Y, Nakamoto Y, Takabayashi A. Usefulness of FDG-positron emission tomography in diagnosing peritoneal recurrence of colorectal cancer. Am J Surg 2002; 184:433-6.

13. Nahmias C, Wahl L. Reproducibility of standardized uptake value measurements determined by $18 \mathrm{~F}-\mathrm{FDG}$ PET in malignant tumors. J Nucl Med 2008; 49:1804-8.
14. Schillaci O. Use of dual-point fluorodeoxyglucose imaging to enhance sensitivity and specificit. Semin Nucl Med 2012; 42:267-80. doi:10.1053/j.semnuclmed.2012.02.003.

15. Roze JF, Hoogendam JP, van de Wetering FT, Spijker R, Verleye $\mathbf{L}$, Vlayen $\mathbf{J}$, et al. Positron emission tomography (PET) and magnetic resonance imaging (MRI) for assessing tumour resectability in advanced epithelial ovarian/fallopian tube/primary peritoneal cancer. Cochrane Database Syst Rev 2018; 10(10):CD012567. doi: 10.1002/14651858.CD012567. pub2.

16. Bushati M, Rovers KP, Sommariva A, Sugarbaker PH, Morris DL, Yonemura Y, et al. The current practice of cytoreductive surgery and HIPEC for colorectal peritoneal metastases: results of a worldwide web-based survey of the Peritoneal Surface Oncology Group International (PSOGI). Eur J Surg Oncol 2018; 44:1942-8.

17. Dromain C, Leboulleux S, Auperin A, Goere D, Malka D, Lumbroso $\mathbf{J}$, et al. Staging of peritoneal carcinomatosis: enhanced CT vs. PET/TC. Abdom Imaging 2008; 33:87-93.

18. Berthelot C, Morel O, Girault S, Verrièle V, Poirier A-L, Moroch J, et al. Use of FDG-PET/TC for peritoneal carcinomatosis before hyperthermic intraperitoneal chemotherapy. Nucl Med Comm 2011; 32:23-9.

19. Whiteford MH, Whiteford HM, Yee LF, Ogunbiyi OA, Dehdashti F, Siegel BA, et al. Usefulness of FDG-PET scan in the assessment of suspected metastatic or recurrent adenocarcinoma of the colon and the rectum. Dis Colon Rectum 2000; 43:767-70.

20. Higashi K, Ueda Y, Seki H, Yuasa K, Oguchi M, Noguchi T, et al. Fluorine-18-FDG PET imaging is negative in bronchioloalveolar lung carcinoma. J Nucl Med 1998; 39:1016-20.

21. Rubini G, Altini C, Notaristefano A, Merenda N, Rubini D, Stabile Ianora AA, et al. Role of 18F-FDG PET/TC in diagnosing peritoneal carcinomatosis in the restaging of patient with ovarian cancer as compared to contrast enhanced CT and tumor marker Ca-125. Rev Esp Med Nucl Imagen Mol 2014; 33:22-7.

22. Li J, Yan R, Lei J, Jiang C. Comparison of PET with PET/CT in detecting peritoneal carcinomatosis: a meta-analysis. Abdom Imaging 2015; 40:2660-6.

23. Schwenzer NF, Schmidt H, Gatidis S, Brendle C, Müller M, Königsrainer I, et al. Measurement of apparent diffusion coefficient with simultaneous MR/positron emission tomography in patients with peritoneal carcinomatosis: comparison with 18F-FDG-PET. J Magn Reson Imaging 2014; 40(5):1121-8.

Contribución de autores
Álvaro Castro, https://orcid.org/0000.0002.0009.0048. Diseño, concepción, análisis y redacción.
Leandro Telles, https://orcid.org/0000.0003.4830.5578. Análisis y redacción.
Gabriela Rodríguez Cantera, https://orcid.org/0000.0002.6076.4999. Análisis y redacción.
Daniel González, https://orcid.org/0000.0003.3916.9201. Concepción y análisis de resultados.
Fernando González, https://orcid.org/0000.0002.6115.0737. Concepción y análisis de resultados.
Luis Ruso Martínez, https://orcid.org/0000.0003.4206.4304. Análisis y revisión crítica. 